\title{
Implementing shared decision making in the NHS: lessons from the MAGIC programme
}

\section{(C) $\mathbb{( \Phi )}$ OPEN ACCESS}

In this analysis article (BMJ 2017;357:j1744, doi:10.1136/bmj. j1744) coauthor Glyn Elwyn was incorrectly affiliated to CK Health Consultancy, Newcastle upon Tyne, UK, when he is actually at the Dartmouth Institute for Health Policy and Clinical Practice, New Hampshire, USA. 